\title{
Dynamics Analysis and Control of a Five-Term Fractional-Order System
}

\author{
Li-xin Yang $\mathbb{D}^{1}$ and Xiao-jun Liu $\mathbb{D}^{2}$ \\ ${ }^{1}$ School of Arts and Sciences, Shaanxi University of Science and Technology, Xian 710021, China \\ ${ }^{2}$ School of Mathematics and Statistics, Tianshui Normal University, Tianshui 741001, China \\ Correspondence should be addressed to Li-xin Yang; jiaodayanglixin@163.com
}

Received 31 May 2018; Revised 22 July 2018; Accepted 10 September 2018; Published 9 October 2018

Guest Editor: Abdul Qadeer Khan

Copyright (C) 2018 Li-xin Yang and Xiao-jun Liu. This is an open access article distributed under the Creative Commons Attribution License, which permits unrestricted use, distribution, and reproduction in any medium, provided the original work is properly cited.

\begin{abstract}
This paper proposes a new fractional-order chaotic system with five terms. Firstly, basic dynamical properties of the fractional-order system are investigated in terms of the stability of equilibrium points, Jacobian matrices theoretically. Furthermore, rich dynamics with interesting characteristics are demonstrated by phase portraits, bifurcation diagrams numerically. Besides, the control problem of the new fractional-order system is discussed via numerical simulations. Our results demonstrate that the new fractional-order system has compound structure.
\end{abstract}

\section{Introduction}

Recently, the study of fractional calculus has attracted great attention due to its potential applications in various fields [13]. As a branch of mathematical analysis, fractional calculus can be considered as the generalization of the conventional calculus. Although the fractional-order derivative theory has a more than 300-year-old history, its application of the theory is just gaining attention [4-6]. In fact, most of the systems in interdisciplinary fields can be described via fractional calculus [7-9]. Moreover, fractional-order model can provide an explicit description and give a further insight into physical process. That is, fractional-order systems can serve as a valuable tool in the modeling of many phenomena. In view of the fact that fractional calculus provides another good way to describe, predict, and control physical systems accurately, it has been applied to control system, physics, and system modeling. Moreover, with the development of interdisciplinary applications, people found that various research fields can be elegantly described with the help of fractional derivatives, such as viscoelastic bodies, quantitative finance, dielectric polarization, electromagnetic waves, and polymer physics.

On the other hand, chaos and its applications have been intensively investigated and developed in many fields of science. In [9], the authors have presented analytical proofs of fold Hopf bifurcation in hyperchaotic Chen system and given sufficient conditions for stability and instability of the bifurcation periodic orbits. Researchers have investigated chaos synchronization of fractional-order systems via linear control [10-12]. Fractional-order systems possess long-range memory behavior and display complexity dynamics characteristic compared to its integral-order counterpart. On the other hand, there exist many significant differences between fractional-order system and the corresponding integer-order differential systems. Generally speaking, fractional-order nonlinear system can display more rich dynamical behaviors such as various bifurcations under certain conditions which are different from the corresponding integer-order system [13, 14]. Several studies have explored the complex dynamical properties in many fractional-order systems, such as fractional-order Chen system [15] and fractional-order Duffing system [16]. Until now, many researchers have investigated the dynamics of several fractional-order chaotic systems and obtained many excellent results [17-19]. In addition, searching for new chaotic systems with fewer terms in autonomous differential equations has been developed with much interest by scientists. In [20], the author has investigated the dynamics of a five-term chaotic attractor. However, this system with fractional order has not been actively and deeply explored, and it is very interesting in a number 
of different fields. To the best of our knowledge, chaotic attractors with fewer than five terms in three fractional-order differential equations have never been investigated.

Motivated by the above, within this body of work, we focus on the dynamical behaviors of this fractionalorder simplified system. This would be of mathematical and practical interests. Rich dynamical behaviors are studied via bifurcation diagrams with varying the system parameters and the fractional derivative orders. Moreover, the control problem of the new fractional system is also investigated.

The remainder of this paper is organized as follows. In Section 2, the definition for the fractional calculus is given. Section 3 is devoted to the investigation of the fractionalorder new system. In Section 4, the control of the new fractional-order system is investigated. Conclusions of this paper will be drawn in Section 5 .

\section{Fractional Calculus}

Fractional calculus can be considered as a generalization of integration and differentiation to a noninteger-order integrodifferential operator ${ }_{a} D_{t}^{q}$ which is described as

$$
{ }_{a} D_{t}^{q}= \begin{cases}\frac{d^{q}}{d t^{q}} & R(q)>0 \\ 1 & R(q)=0 \\ \int_{a}^{t}(d \tau)^{-q} & R(q)<0\end{cases}
$$

where $q$ denotes derivative order and $R(q)$ corresponds to the real part of $q$. The numbers $a$ and $t$ represent the limits of the operator. At present, there are several definitions of the fractional-order differential system. Riemann-Liouville and Caputo definitions are considered the most common and efficient fractional derivatives [21,22].

Firstly, the Riemann-Liouville (RL) definition of fractional derivatives can be written as follows.

$$
\begin{aligned}
{ }_{a}^{R L} D_{t}^{q} f(t)=\frac{d^{n}}{d t^{n}} \frac{1}{\Gamma(n-q)} \int_{a}^{t} \frac{f(\tau)}{(t-\tau)^{q-n+1}} d \tau, & \\
& n-1<q<n
\end{aligned}
$$

The Caputo fractional derivative is given by the following.

$$
\begin{array}{r}
{ }_{a}^{C} D_{t}^{q} f(t)=\frac{1}{\Gamma(n-q)} \int_{a}^{t}(t-\tau)^{n-q-1} f^{(n)}(\tau) d \tau, \\
n-1<q<n
\end{array}
$$

In the above formulations, $\Gamma(\cdot)$ represents the Gamma function.

$$
\Gamma(s)=\int_{0}^{\infty} t^{s-1} e^{-t} d t .
$$

Note that the Caputo derivative guarantees a straightforward connection between the types of the initial condition and the fractional derivative. Hence, the Caputo derivative is adopted in this paper.

\section{An Unusual Five-Term Fractional-Order System}

At first, a simple chaotic integer-order system with five terms is described as follows [20]:

$$
\begin{aligned}
& \dot{x}=a(y-x) \\
& \dot{y}=-x z \\
& \dot{z}=-b+x y,
\end{aligned}
$$

when the parameters are selected as $a=5, b=90$, system (5) consists of two quadratic nonlinearities and displays abundantly complex behaviors of chaotic dynamics.

In what follows, we suppose that the derivative orders are fractional; the equations of the fractional-order system are readily derived from the above integer-order counterpart

$$
\begin{aligned}
& D_{*}^{q_{1}} x=a(x-y) \\
& D_{*}^{q_{2}} y=-x z \\
& D_{*}^{q_{3}} z=-b+x y,
\end{aligned}
$$

where $q_{i}(i=1,2,3)$ are the fractional derivatives orders.

In the next step, the dynamical behaviors of this new fractional-order system are investigated.

3.1. Some Properties of the New Fractional-Order System. It should be noted that most of the theory for the integerorder dynamic system cannot be simply extended to the fractional-order system. Therefore, the sufficient conditions of the stability of the fractional-order systems are given [23].

Lemma 1. An autonomous fractional-order system is asymptotically steady at the equilibrium, if all the eigenvalues of the Jacobian matrix of some equilibrium satisfy

$$
|\arg (\operatorname{eig}(J))|>\frac{q \pi}{2}, \quad q=\max \left(q_{1}, q_{2}, q_{3}\right),
$$

where eig $(J)$ denotes the eigenvalues of matrix $J$.

Based on the above theorem, the equilibria of system (6) can be calculated by solving the following equations

$$
\begin{aligned}
& D_{*}^{q_{1}} x=a(x-y)=0 \\
& D_{*}^{q_{2}} y=-x z=0 \\
& D_{*}^{q_{3}} z=-b+x y=0 .
\end{aligned}
$$

The system contains two equilibrium points, i.e.,

$$
\begin{aligned}
P^{+}(x, y, z) & =(+\sqrt{b},+\sqrt{b}, 0), \\
P^{-}(-x,-y,-z) & =(-\sqrt{b},-\sqrt{b}, 0),
\end{aligned}
$$

and, for the equilibrium $P^{+}(x, y, z)=(+\sqrt{b},+\sqrt{b}, 0)$, the Jacobian matrix of (6) at points $P^{+}$is obtained as

$$
J^{+}=\left[\begin{array}{ccc}
-a & a & 0 \\
-z & 0 & -x \\
y & x & 0
\end{array}\right]=\left[\begin{array}{ccc}
-a & a & 0 \\
0 & 0 & -\sqrt{b} \\
\sqrt{b} & \sqrt{b} & 0
\end{array}\right] .
$$


Then we can obtain the eigenvalues of the Jacobian matrix as follows.

$$
\begin{aligned}
& \lambda_{1}=-7.0943 \\
& \lambda_{2}=1.4701+10.5444 i \\
& \lambda_{3}=1.4701-10.5444 i
\end{aligned}
$$

For the second equilibrium $P^{-}(x, y, z)=(-\sqrt{b},-\sqrt{b}, 0)$, the Jacobian matrix of the fractional-order new system (6) is defined as

$$
J^{-}=\left[\begin{array}{ccc}
-a & a & 0 \\
-z & 0 & -x \\
y & x & 0
\end{array}\right]=\left[\begin{array}{ccc}
-a & a & 0 \\
0 & 0 & \sqrt{b} \\
-\sqrt{b} & -\sqrt{b} & 0
\end{array}\right] .
$$

By computing $|\lambda E-J|=0$, it is found that the eigenvalues of the Jacobian matrix $J^{-}$are the same as those of the Jacobian matrix $J^{+}$. That is, $\lambda_{1}$ is a negative real number and $\left|\arg \left(\lambda_{2,3}\right)\right|=1.4323$; then according to Lemma 1, the eigenvalues cannot satisfy (7) as $0.912 \leq q \leq 1.0$, and two equilibrium points of the nonlinear fractional-order system (6) are all unstable.

3.2. Chaos and Bifurcations with Fractional-Order Parameters. To investigate the existence of new attractors in the fractionalorder system, this part is devoted to dynamics of system (6) by considering several values of the fractional derivatives orders.

First, system parameters are selected as $a=5, b=90$, and the order parameter $q_{i}(i=1,2,3)$ is varied. Figure 1 shows several typical attractors for $q_{1}=q_{2}=q_{3}=\theta$; in this case, the fractional-order system is a commensurate-order system. The initial states of the new fractional-order system are taken as $x(0)=1, y(0)=2, z(0)=3$. From these figures, one can observe that the new fractional-order system exhibits rich dynamical behaviors.

When the derivative order $\theta=0.84$ and $\theta=0.86$, system (6) stabilizes to an equilibrium point, as shown in Figures 1(a) and $1(\mathrm{~b})$. When $\theta$ further increases to 0.88 , there exists a single attractor, as reflected in Figure 1(c). The system also shows a chaotic attractor for $\theta=0.98$, but it is different from the case of $\theta=0.88$, as shown in Figure $1(\mathrm{~d})$.

In order to further study the complex dynamics of the fractional-order system, consequently, the bifurcation diagrams with fractional derivative orders are given. First of all, we focus on the case of commensurate-order system. The fractional derivative order $q$ varies from 0.88 to 1 . Figure 2 represents the bifurcation diagram of the fractional-order system. It is clearly shown that the new fractional-order system is chaotic over most of the scopes $q \in[0.89,0.95]$ and $q \in[0.96,1]$. There exist tangent bifurcations when parameter $q<0.96$. And the fractional-order system enters into chaos again as $q>0.96$.

Phase portraits are shown in Figures 3(a) and 3(b); from the two figures, it can be clearly seen that the system shows distinguishable dynamical behaviors for different values of $q$.

As we know, for the incommensurate-order system, dynamical behavior is more complex than the commensurate-order system. Therefore, here, several typical differential order values are selected. We focus our attention on bifurcations versus the different derivative orders.

Fix the fractional derivative orders $q_{2}=q_{3}=0.98$; dynamical behavior of system (6) with fractional derivative order $q_{1} \in[0.91,0.99]$ is presented as Figure 4 . With increasing of the order parameter $q_{1}$ from 0.91 , the fractionalorder system enters into chaos by a series of period-doubling bifurcations. It can be seen that the system is period-2 for $q=0.92$, period -4 for $q=0.93$. The route out of chaos for the system is tangent bifurcation. Also, it can be observed that saddle-node bifurcation occurs when $q_{1} \in$ [0.975 0.983]. In the next step, Figure 5 represents the bifurcation diagrams with $q_{2}, q_{3}$ varying by numerical simulation. It is observed that there exist tangent bifurcations for two parameters, but the values of $q_{2}, q_{3}$ are different. The route out of chaos for the system is through tangent bifurcation when $q_{2}<0.92$, but transient chaos is observed when $q_{3}<$ 0.96 .

3.3. Chaos and Bifurcations with System Parameters. First, fix fractional derivative orders $q_{1}=q_{2}=q_{3}=0.98$, and let system parameter $a$ vary. The parameter $a$ varies from 5 to 8 . The bifurcation diagram with parameter $a$ is shown in Figure 6. System displays chaotic behavior when parameter $a<5.2$, as shown in Figure 7(a). When bifurcation parameter $a$ increases from 6 to 6.5, there exist a series of period-doubling bifurcations, such as period-1 and period- 4 [see Figures 7(b) and 7(c)]. If $a$ keeps increasing, bifurcation occurs and the system switches to a periodic motion, as Figure $7(\mathrm{~d})$ shows.

To furthermore exhibit the bifurcation behavior of the system (6), the expanded periodic window a $\in\left[\begin{array}{l}6.6 \\ 7.6\end{array}\right]$ and [5.8 6.2] are plotted in Figures 6(b) and 6(c). From these figures, it can be seen that period bifurcation and Hopf bifurcation happen under certain parameters. And different attractors are shown in Figures $7(a)-7(d)$.

In what follows, let the fractional derivative orders $q_{1}=$ $q_{2}=q_{3}=0.91$, system parameter $a=5$; the dynamical behaviors of the system (6) with the variation of parameter $b$ are discussed. Numerically we calculate the values of the parameter $b$; the dynamical behaviors of (6) can be classified as follows:

(i) When $b \leq 175$, system becomes period orbit, as shown in Figure 8(a).

(ii) When $175<b<193$, system displays a limit cycle, as shown in Figure 8(b).

(iii) When $b>193$, system demonstrates a chaotic attractor, as shown in Figure 8(c).

It can be seen that the chaotic attractors structure changes qualitatively with the variation of the parameter $b$ and the order $q$. When the parameter $b$ increases from 175 to 200 , the dynamics of system (6) becomes more and more rich.

\section{Control of New Fractional-Order System}

In this subsection, the control problem of commensurateorder fractional-order system is investigated. For simplicity, we assume the derivative order $q=0.96$, a control parameter 


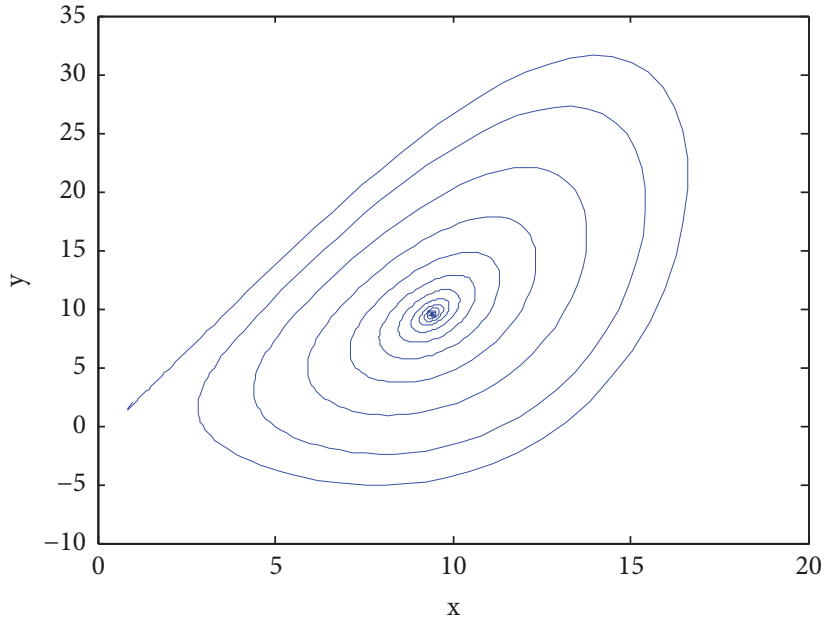

(a)

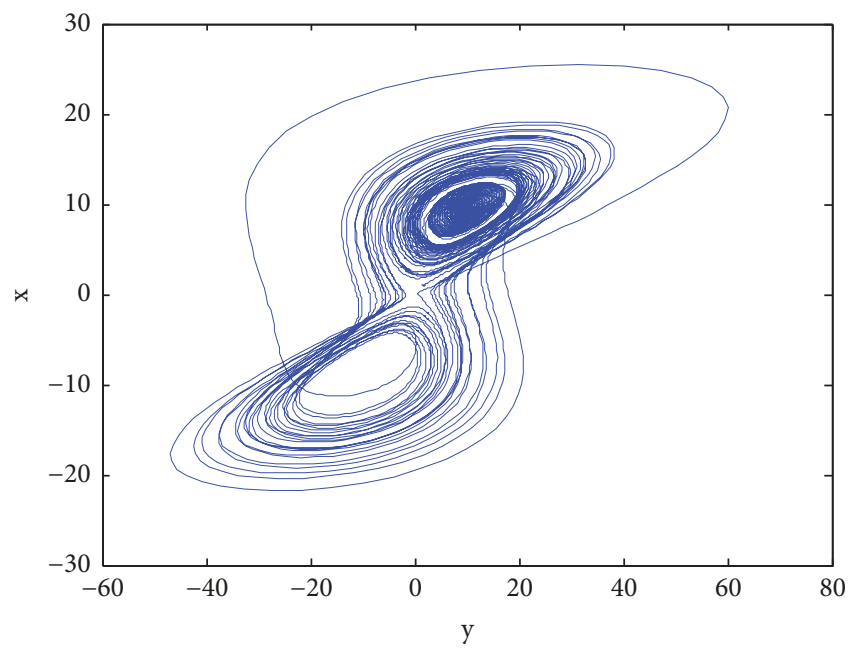

(c)

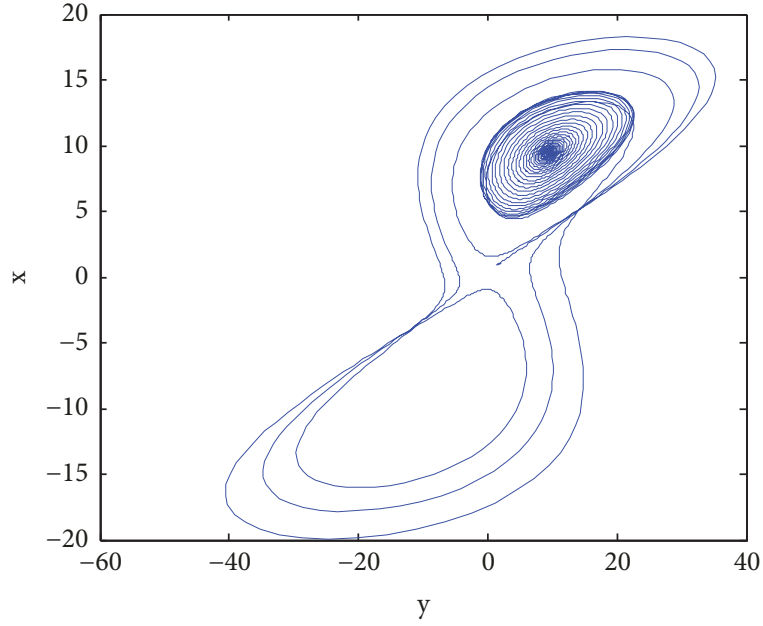

(b)

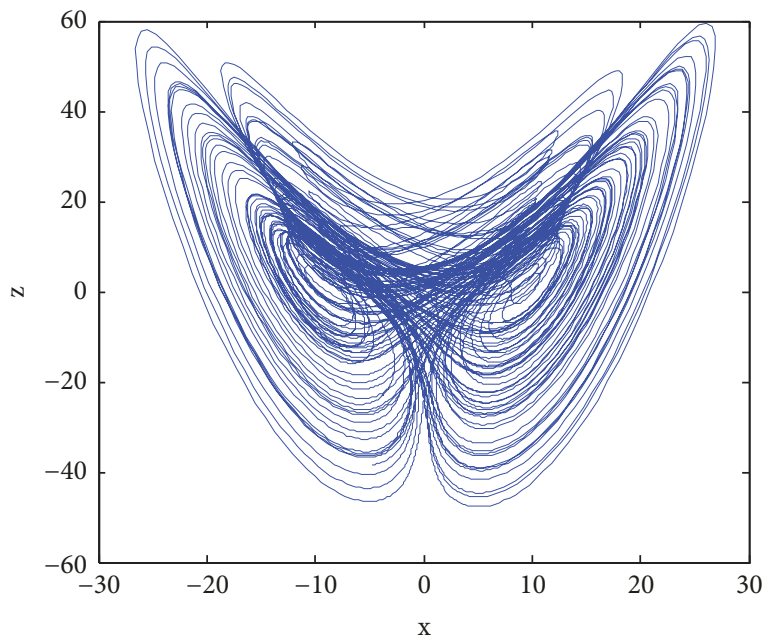

(d)

FIgURE 1: Attractors of system (6) for $q_{1}=q_{2}=q_{3}=\theta$. (a) $\theta=0.84$; (b) $\theta=0.86$; (c) $\theta=0.88$; (d) $\theta=0.98$.

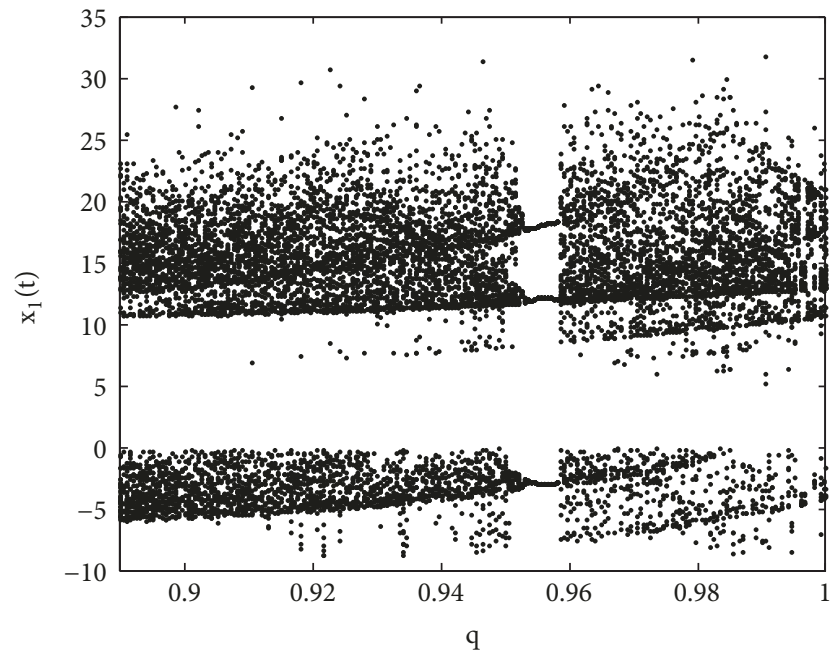

FIGURE 2: Bifurcation diagram of the fractional-order system (6) with $q \in(0.88,1)$. $u$ is added to the second equation of system (6), and the controlled system can be written as

$$
\begin{aligned}
& D_{*}^{q} x=a(x-y) \\
& D_{*}^{q} y=-x z+u \\
& D_{*}^{q} z=-b+x y .
\end{aligned}
$$

Here, we use the predictor-corrector method to obtain the numerical solution of system (13). In our simulations, the time step and the running time are taken as $h=0.001$ and $T=100 \mathrm{sec}$, respectively. The initial conditions of fractionalorder system are selected as $x(0)=y(0)=z(0)=1.0$. If the control parameter $u=-70$, one can obtain the left half-image of the original chaotic attractor (Figure $1(\mathrm{~d})$ ), as shown in Figure 9(a) for the $x-z$ plane. However, when the control parameter $u=70$, the right half-image of the original chaotic attractor can be isolated as shown in Figure 9(b). It implies that the new fractional-order system (6) consists of compound structures under certain conditions. 


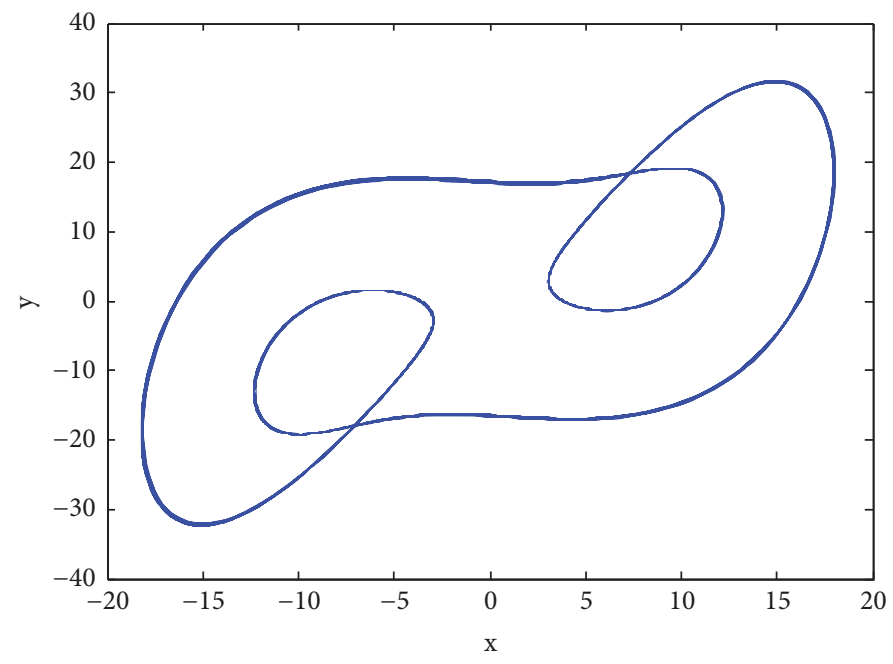

(a)

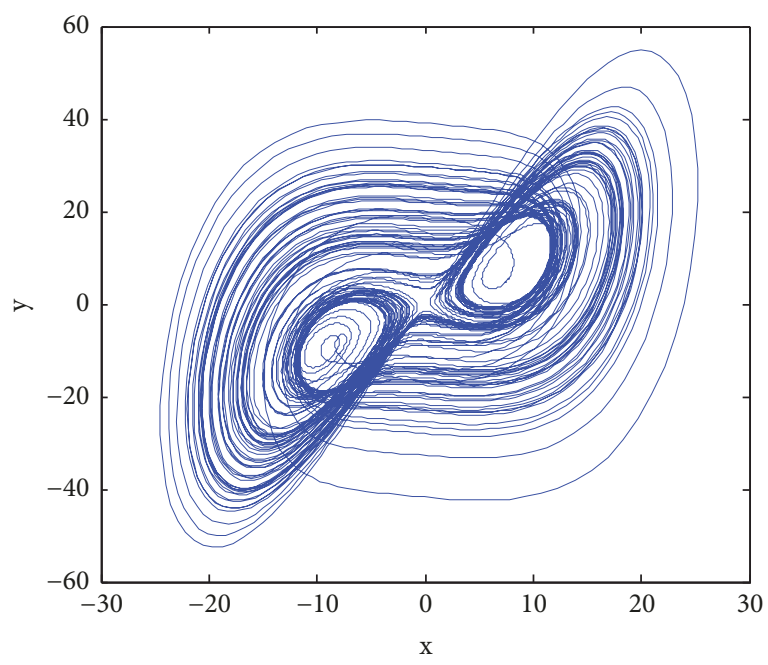

(b)

Figure 3: The phase portraits of the system for different values of $q$ (a) $q=0.95$; (b) $q=0.98$.

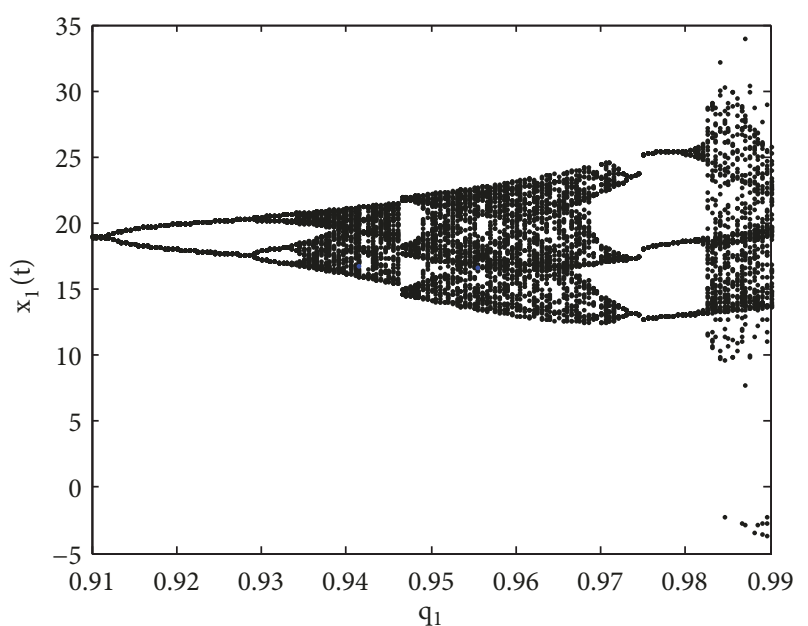

FIGURE 4: Bifurcation diagram of the fractional-order system (6) with $q_{1} \in(0.91,0.99)$.

In the following, fix system parameter $a=5, b=90$, and the fractional derivatives order $q=0.98$; then we adjust the values of the control parameter $u$; complex dynamical behaviors of fractional-order system (13) can be summarized as follows:

(i) When $98 \leq|u| \leq 170$, system (13) displays limit cycles. For example, Figure 10(a) demonstrates a limit cycle at $u=98$.

(ii) When $80 \leq|u| \leq 90$ and $71 \leq|u| \leq 80$, system (13) displays period-doubling bifurcations. Figures 10(b) and 10(c) give different period dynamics.

(iii) When $65 \leq|u| \leq 69$, system (13) exhibits partial attractors. Figure $10(\mathrm{~d})$ shows a partially left, dominantly right attractor.

(iv) When $|u|=70$, system (13) shows left and right halfimage attractors, respectively, as shown in Figures 9(a) and 9(b).

Our results demonstrate that there are different formats of chaos with the variation of derivative order. One is a process of period-doubling bifurcations and the other is an interior crisis from single-scroll to double-scroll attractors. Certainly, the minimum effective dimension for fractional-order system to keep chaos is different between commensurate-order and incommensurate-order case. In order to know more about the dynamics of the system and to make further study in the future, the dynamics of system (6) with the variation of the different derivative order in the incommensurate-order case will be investigated in future work. In addition, future work on the topic should include the analysis of chaos control of the fractional-order system in detail.

\section{Results and Discussion}

In this paper, a novel fractional-order system is presented. Complex dynamics with interesting characteristics of the new fractional-order system are studied. Firstly, the basic properties of the new fractional-order system are analyzed via theoretical scheme. In addition, the phase diagrams for the different values of the parameters are obtained to show the rich dynamics of the system. Furthermore, the bifurcations and chaos dynamical phenomena in this system are numerically investigated by varying the fractional derivative orders and system parameters, respectively. The new fractionalorder system displays several typical bifurcations, such as tangent bifurcations, period bifurcations, and various chaotic attractors. Meanwhile, the control problem of fractionalorder system is investigated. Moreover, forming mechanisms of compound structures of the new fractional-order system is demonstrated via numerical simulations.

\section{Appendix}

\section{A Brief Explanation regarding the Predictor-Corrector Algorithm}

There are two approximation methods which can be used for numerical computation on chaos with fractional differential 


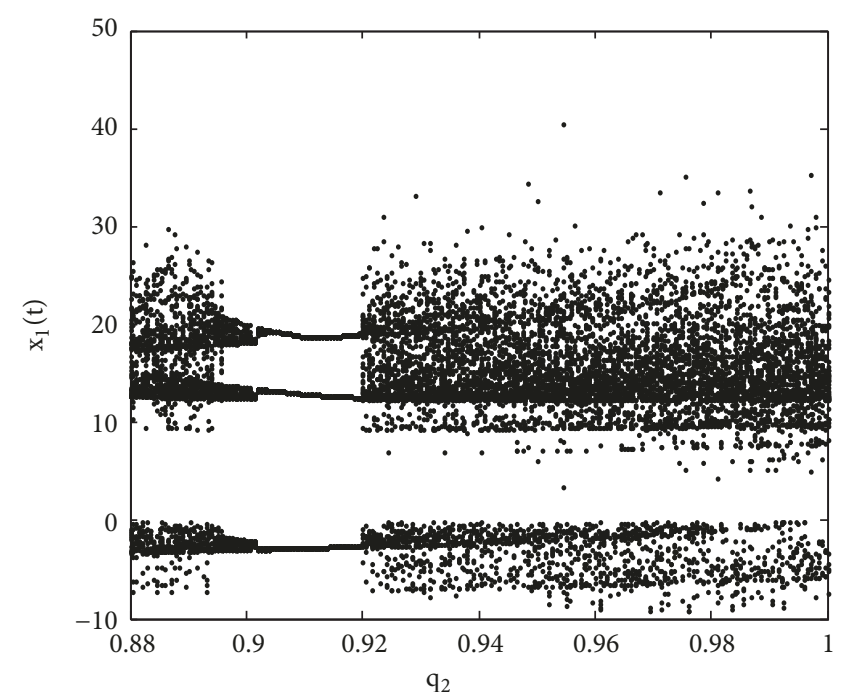

(a)

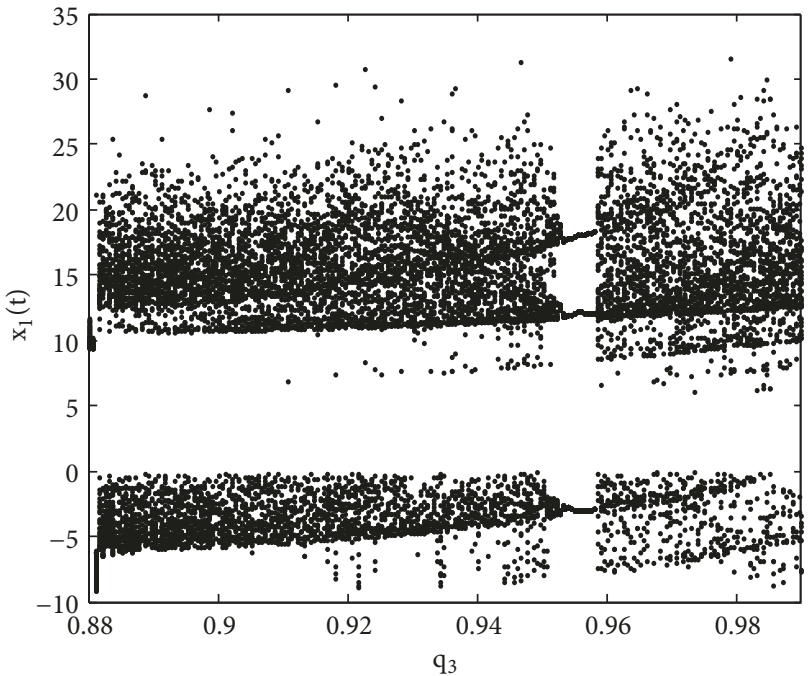

(b)

FIgURE 5: Bifurcation diagram of the fractional-order system (6) with (a) $q_{2} \in(0.88,1)$ (b) $q_{3} \in(0.88,0.99)$.

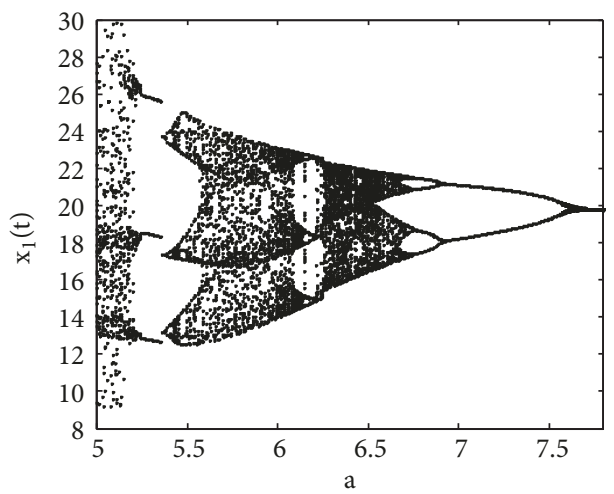

(a)

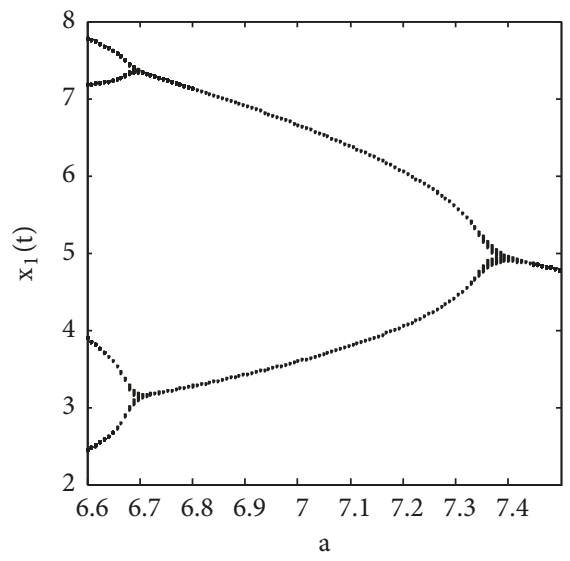

(b)

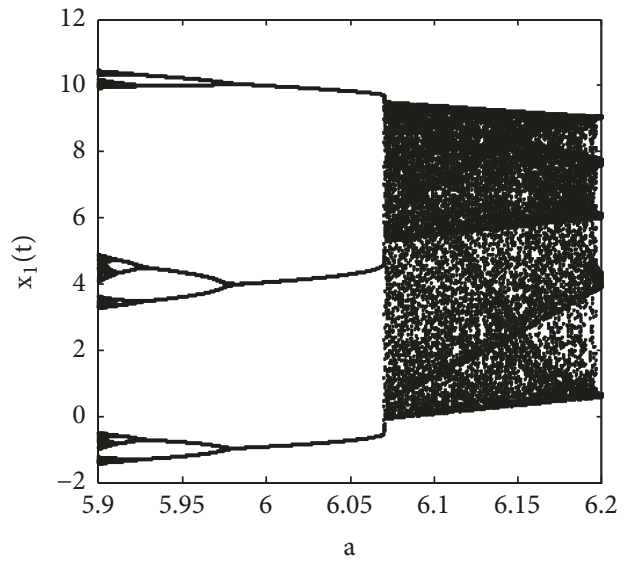

(c)

FIgURE 6: Bifurcation diagram of the fractional-order system (6) versus parameter a. (a) $a \in(5,7.8)$; (b) $a \in(6.6,7.5)$; (c) $a \in(5.8,6.2)$.

equations. One is an improved version of Adams-BashforthMoulton algorithm based on the predictor-correctors scheme, which is a time-domain approach. The other is frequency domain approximation, based on numerical analysis of fractional-order systems in the frequency domain. In this paper, we employ the improved predictor-corrector algorithm for fractional-order differential equations. In what follows, the predictor-corrector algorithm is introduced. 


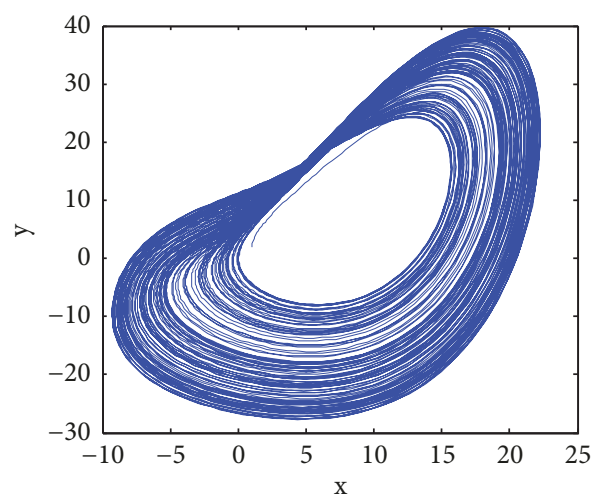

(a)

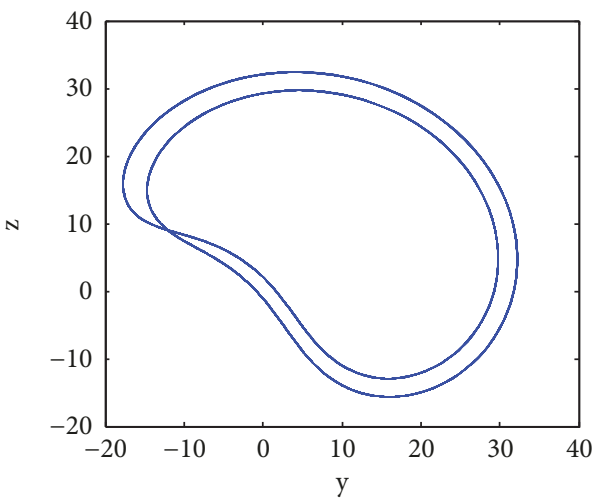

(c)

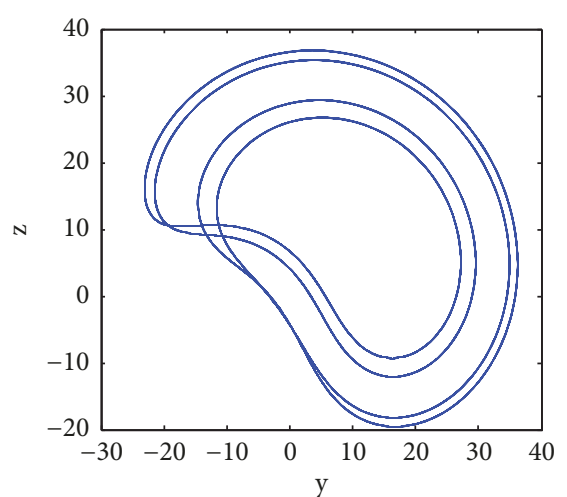

(b)

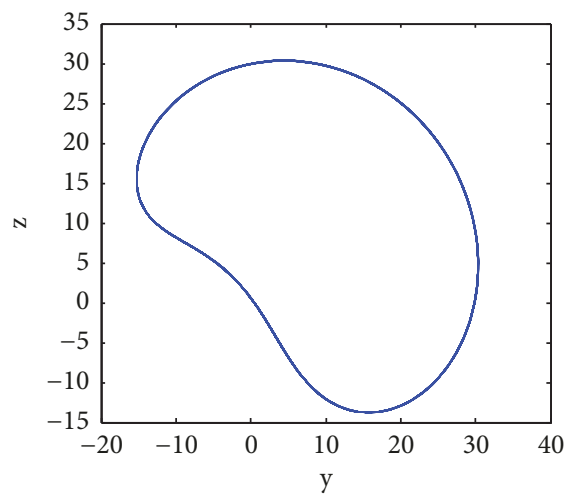

(d)

Figure 7: Phase portraits of the fractional-order system (6) for $q_{i}(i=1,2,3)=0.98$. (a) $a=5.2$; (b) $a=6.8$; (c) $a=7.3$; (d) $a=7.8$.

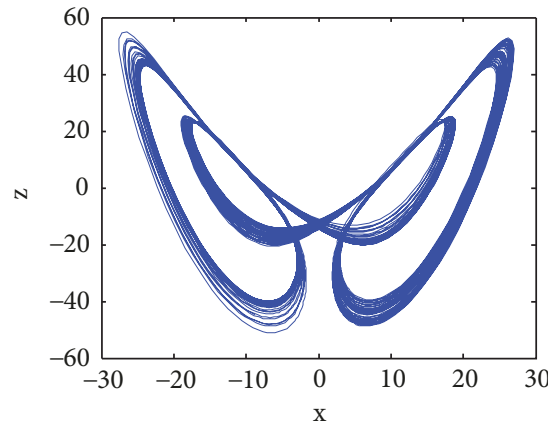

(a)

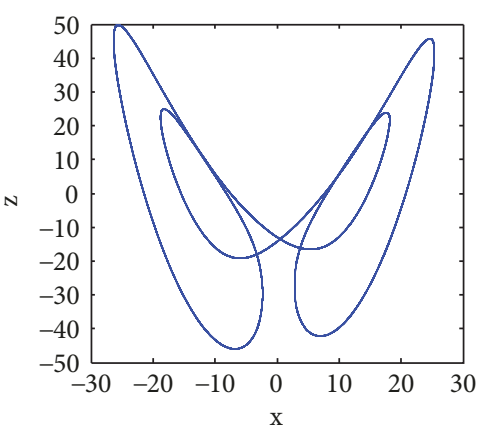

(b)

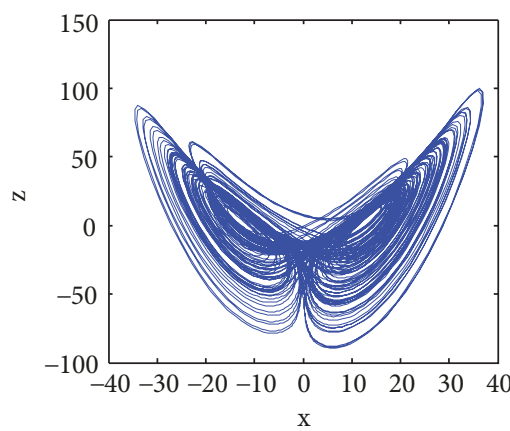

(c)

FIGURE 8: The phase portraits for system (6) at (a) $b=175$; (b) $b=180$; (c) $b=200$.

To get the approximate solution of a fractional-order chaotic system by the predictor-corrector algorithm, the following equation is investigated

$$
\begin{gathered}
\frac{d^{q} x}{d t^{q}}=f(t, x) \quad 0 \leq t \leq T \\
x^{k}(0)=x_{0}^{k} \quad k=0,1, \ldots,[q]-1,
\end{gathered}
$$

which is equivalent to the Volterra integral equation.

$$
x(t)=\sum_{k=0}^{[\alpha]-1} \frac{t^{k}}{k !} x_{0}^{(k)}
$$

$$
+\frac{1}{\Gamma(\alpha)} \int_{0}^{t}(t-\tau)^{\alpha-1} f(\tau, x(\tau)) d \tau
$$

Set $h=T / N, t_{j}=j h,(j=0,1, \ldots, N)$. Then the corrector formula for (2) can be discretized as follows:

$$
\begin{aligned}
x_{h}\left(t_{n+1}\right)= & \sum_{k=0}^{[\alpha]-1} \frac{t_{n+1}^{k}}{k !} x_{0}(k) \\
& +\frac{h^{\alpha}}{\Gamma(\alpha+2)} f\left(t_{n+1}, x_{h}^{p}\left(t_{n+1}\right)\right)
\end{aligned}
$$




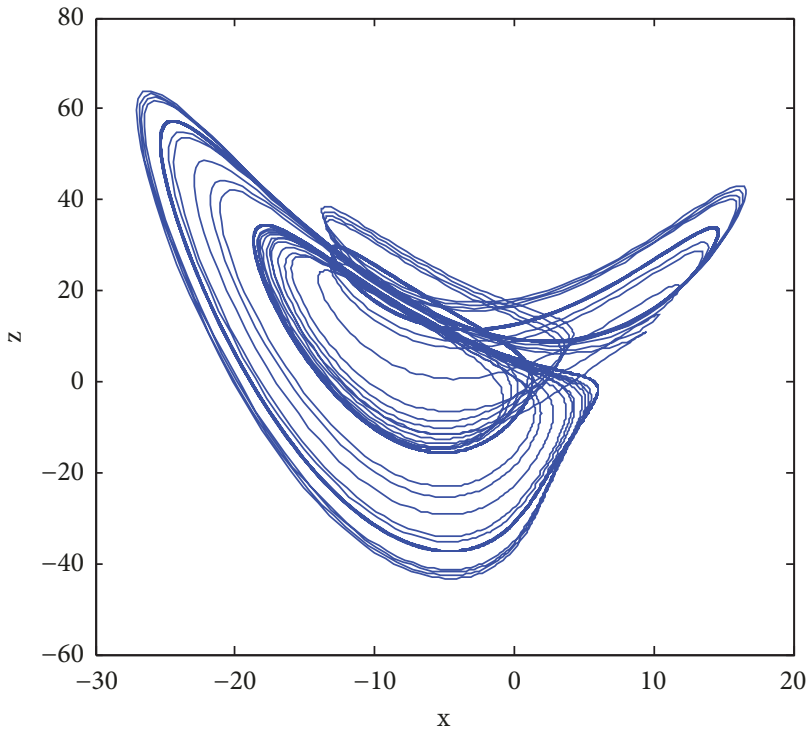

(a)

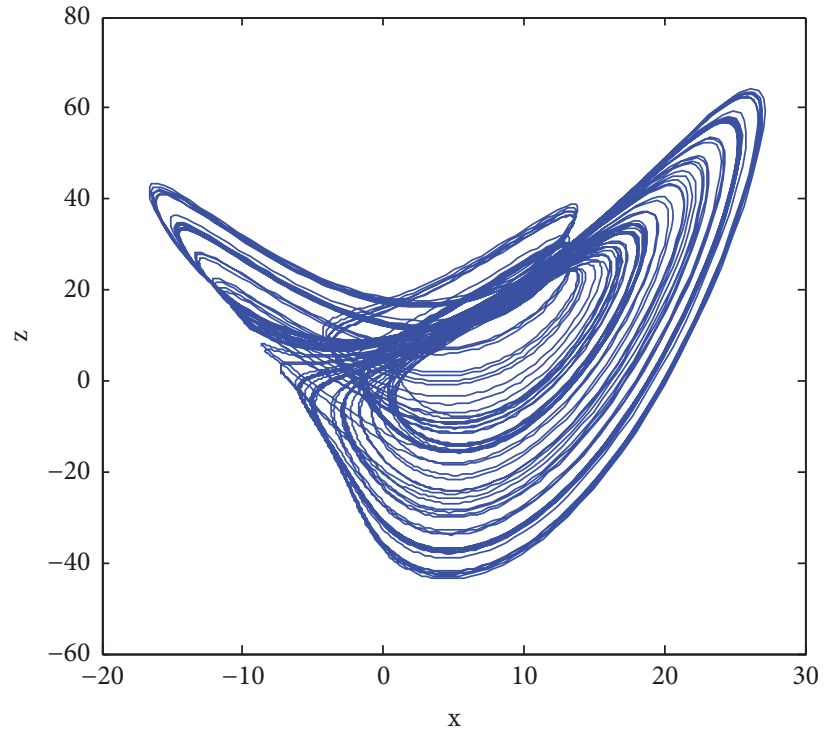

(b)

Figure 9: (a) Left half-image attractor for $u=-70$ (b) right half-image attractor for $u=70$.

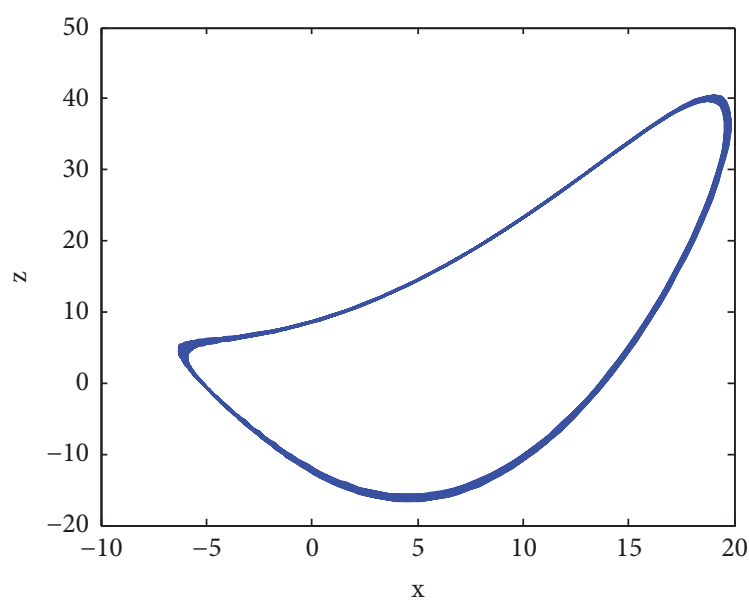

(a)

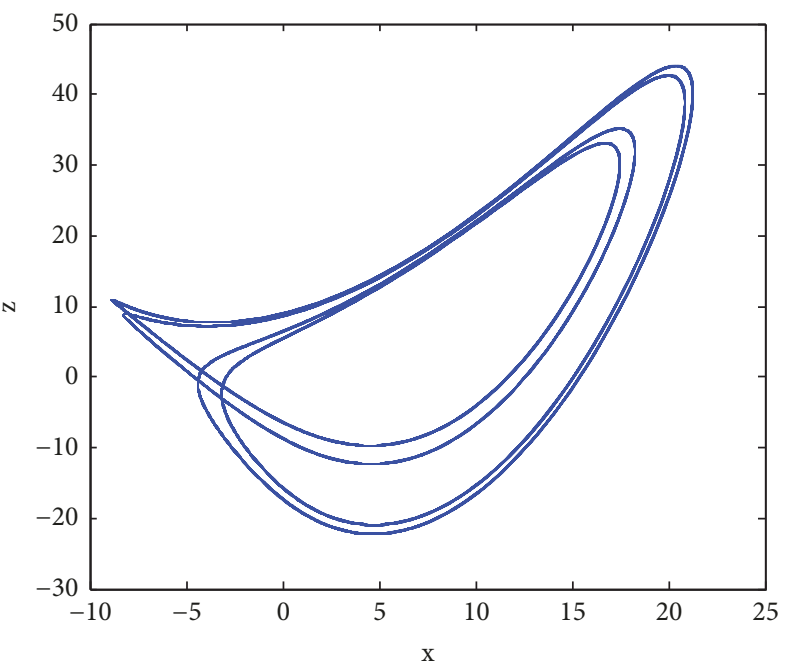

(c)

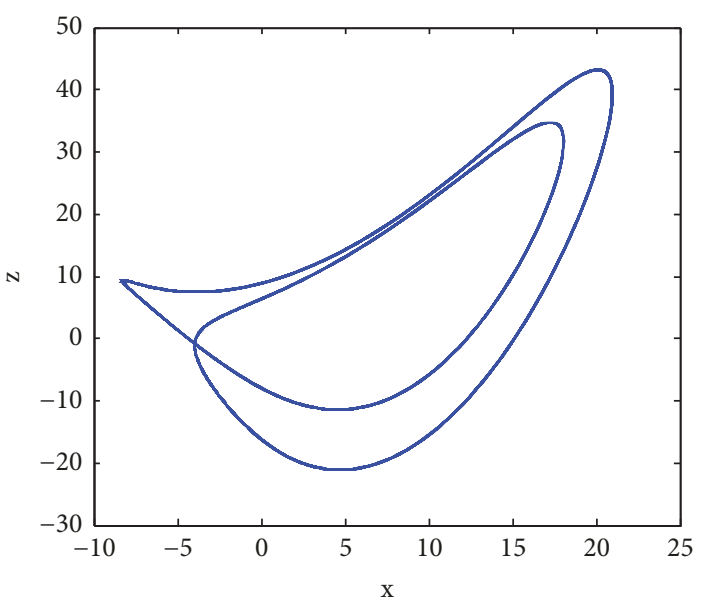

(b)

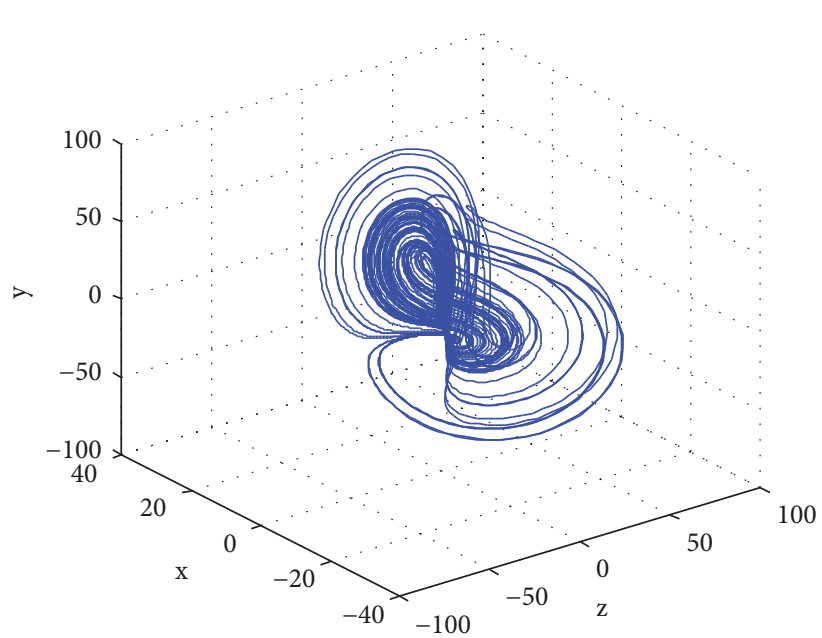

(d)

FIGURE 10: Attractors of system (13) at (a) $u=98$; (b) $u=90$; (c) $u=88$; (d) $u=68$. 


$$
+\frac{h^{\alpha}}{\Gamma(\alpha+2)} \sum a_{j, n+1} f\left(t_{j}, x_{h}\left(t_{j}\right)\right)
$$

where predicted values $x_{h}\left(t_{n+1}\right)$ are determined by the following formula.

$$
\begin{aligned}
x_{h}^{p}\left(t_{n+1}\right)= & \sum_{k=0}^{[q]-1} x_{0}^{(k)} \frac{t_{n+1}^{k}}{k !} \\
& +\frac{1}{\Gamma(q)} \sum_{j=0}^{n} \beta_{j, n+1} f\left(t, x_{h}\left(t_{j}\right)\right),
\end{aligned}
$$

Also

$$
\begin{aligned}
& \alpha_{j, n+1} \\
& = \begin{cases}n^{q+1}-(n-q)(n+1)^{q}, & j=0 \\
(n-j+2)^{q+1}+(n-j)^{q+1}-2(n-j+1)^{q+1}, & 1 \leq j \leq n \\
1, & j=n+1 .\end{cases} \\
& \beta_{j, n+1}=\frac{h^{q}}{q}\left((n-j+1)^{q}-(n-j)^{q}\right), \quad 1 \leq j \leq n .
\end{aligned}
$$

The error estimate of this approach can be presented: $\max _{j=0,1, \ldots, N}\left|x\left(t_{j}\right)-x_{h}\left(t_{j}\right)\right|=O\left(h^{P}\right)$, where $p=\min (2,1+q)$.

\section{Data Availability}

Data used to support the findings of this study are included within the paper.

\section{Conflicts of Interest}

The authors declare that they have no conflicts of interest.

\section{Acknowledgments}

This work is supported by the National Natural Science Foundation of China (NSFC) under grant No. 11702195 and No. 11702194 and Natural Science Preparatory Study Foundation of Shaanxi University of Science and Technology under grant No. 2017 BJ-49.

\section{References}

[1] C. P. Li, W. H. Deng, and D. Xu, "Chaos synchronization of the Chua system with a fractional order," Physica A: Statistical Mechanics and its Applications, vol. 360, no. 2, pp. 171-185, 2006.

[2] J. Huo and H. Zhao, "Dynamical analysis of a fractional SIR model with birth and death on heterogeneous complex networks," Physica A: Statistical Mechanics and its Applications, vol. 448, pp. 41-56, 2016.

[3] G. M. Mahmoud, T. Bountis, G. M. AbdEl-Latif, and E. E. Mahmoud, "Chaos synchronization of two different chaotic complex Chen and Lü systems," Nonlinear Dynamics, vol. 55, no. 1-2, pp. 43-53, 2009.
[4] S. Wang, Y. Yu, and G. Wen, "Hybrid projective synchronization of time-delayed fractional order chaotic systems," Nonlinear Analysis: Hybrid Systems, vol. 11, pp. 129-138, 2014.

[5] X. F. Li, Y. D. Chu, J. G. Zhang, and Y. X. Chang, "Nonlinear dynamics and circuit implementation for a new autonomous chaotic system," Chaos Solitions and Fractals, vol. 41, no. 5, pp. 2360-2370, 2009.

[6] S.-J. Ma, Q. Shen, and J. Hou, "Modified projective synchronization of stochastic fractional order chaotic systems with uncertain parameters," Nonlinear Dynamics, vol. 73, no. 1-2, pp. 93-100, 2013.

[7] Z. M. Odibat, "Adaptive feedback control and synchronization of non-identical chaotic fractional order systems," Nonlinear Dynamics, vol. 60, no. 4, pp. 479-487, 2010.

[8] H.-Y. Jia, Z.-Q. Chen, and W. Xue, "Analysis and circuit implementation for the fractional-order Lorenz system," Acta Physica Sinica, vol. 62, no. 14, Article ID 140503, 2013.

[9] X. Wang and H. Zhang, "Bivariate module-phase synchronization of a fractional-order lorenz system in different dimensions," Journal of Computational and Nonlinear Dynamics, vol. 8, no. 3, p. 031017, 2013.

[10] C. Li and G. Chen, "Chaos and hyperchaos in the fractionalorder Rössler equations," Physica A: Statistical Mechanics and its Applications, vol. 341, no. 1-4, pp. 55-61, 2004.

[11] J.-L. Lavoie, T. J. Osler, and R. Tremblay, "Fractional derivatives and special functions," SIAM Review, vol. 18, no. 2, pp. 240-268, 1976.

[12] L. Kocarev and U. Parlitz, "General approach for chaotic synchronization with applications to communication," Physical Review Letters, vol. 74, no. 25, pp. 5028-5031, 1995.

[13] G. M. Mahmoud and T. Bountis, "The dynamics of systems of complex nonlinear oscillators: a review," International Journal of Bifurcation and Chaos, vol. 14, no. 11, pp. 3821-3846, 2004.

[14] B. Vinagre and V. Feliu, "Modeling and control of dynamic systems using fractional calculus: application to electrochemical processes and flexible structures," in Proceedings of the 41st IEEE Conference on Decision and Control, pp. 214-239, 2002.

[15] Z. M. Ge and C. Y. Ou, "Chaos in a fractional order modified Duffing system," in Proceedings of the ECCTD, pp. 1259-1262, 2005.

[16] J. G. Lu and G. R. Chen, "A note on the fractional-order Chen system," Chaos Solitions and Fractals, vol. 27, no. 3, pp. 685-688, 2006.

[17] X. Wu, H. Wang, and H. Lu, "Modified generalized projective synchronization of a new fractional-order hyperchaotic system and its application to secure communication," Nonlinear Analysis: Real World Applications, vol. 13, no. 3, pp. 1441-1450, 2012.

[18] Y. Kuang, Delay Differential Equations with Applications in Population Dynamics, Academic Press, New York, NY, USA, 1993.

[19] J.-G. Lu, "Chaotic dynamics of the fractional-order Ikeda delay system and its synchronization," Chinese Physics, vol. 15, no. 2, pp. 301-305, 2006.

[20] B. Munmuangsaen and B. Srisuchinwong, "A new five-term simple chaotic attractor," Physics Letters A, vol. 373, no. 44, pp. 4038-4043, 2009.

[21] S. Bhalekar, "Dynamical analysis of fractional order Uçar prototype delayed system," Signal, Image and Video Processing, vol. 6, no. 3, pp. 513-519, 2012. 
[22] I. Podlubny, Fractional Differential Equations, vol. 198 of Mathematics in Science and Engineering, Academic Press, San Diego, Calif, USA, 1999.

[23] D. Matignon, "Stability results for fractional differential equations with applications to control processing," in Proceedings of the IEEE-SMC Proceedings Computational engineering in systems and application multi-conference, vol. 2, pp. 963-968, IMACS, Lille, France, 1996. 


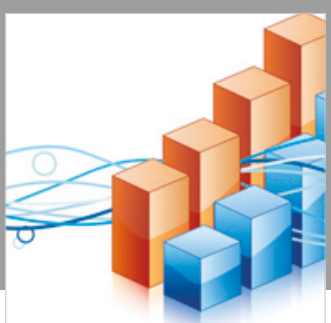

Advances in

Operations Research

\section{-n-m}
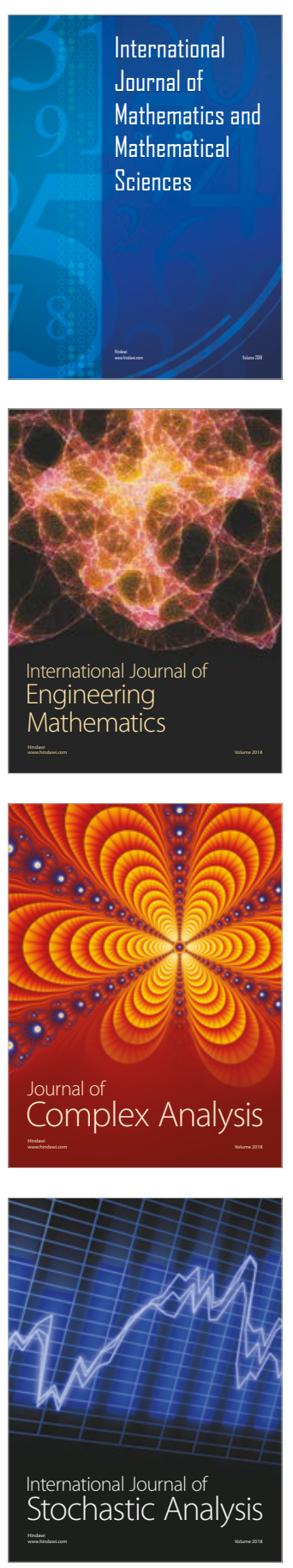
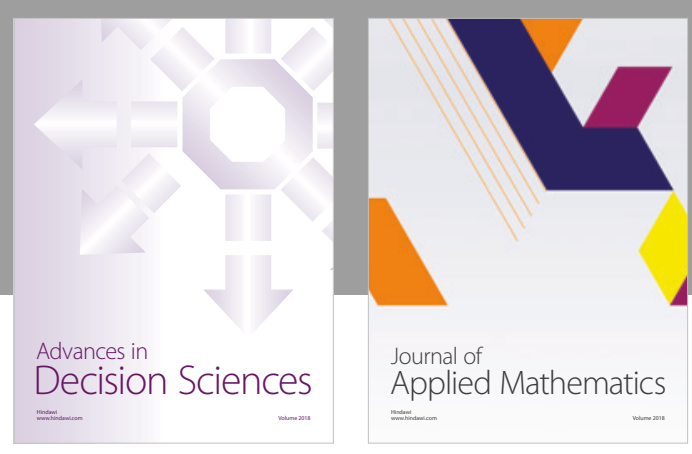

Journal of

Applied Mathematics
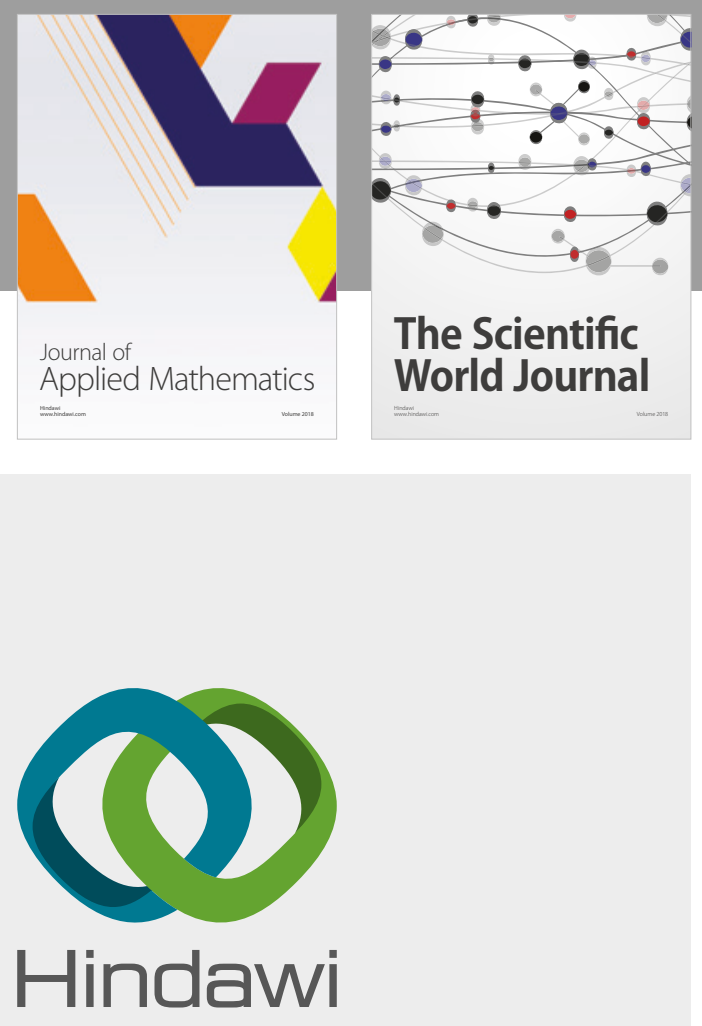

Submit your manuscripts at

www.hindawi.com

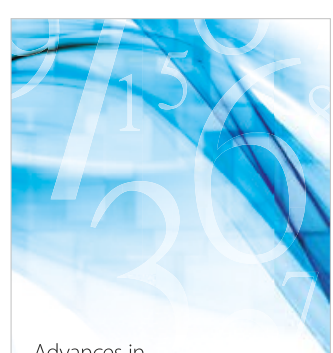

Advances in
Numerical Analysis
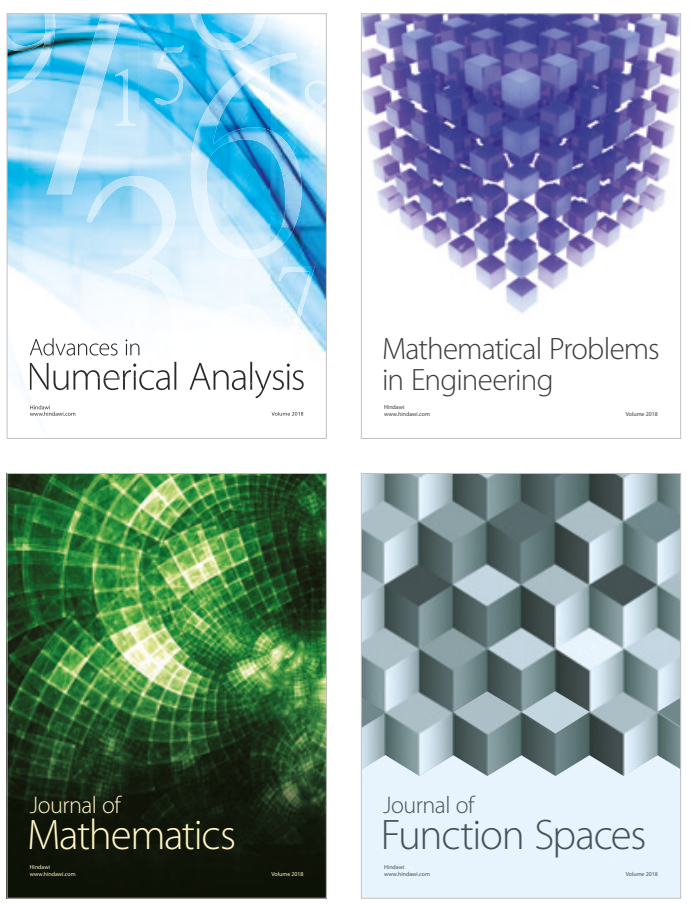

Mathematical Problems in Engineering

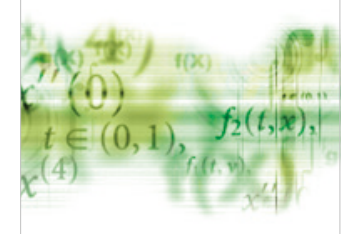

International Journal of

Differential Equations

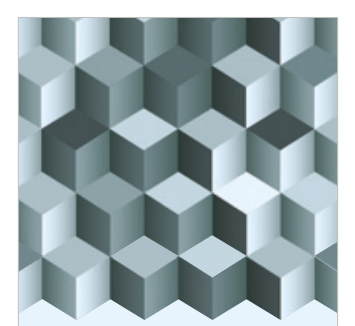

Journal of

Function Spaces

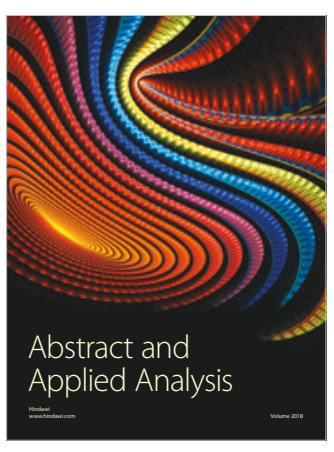

The Scientific

World Journal

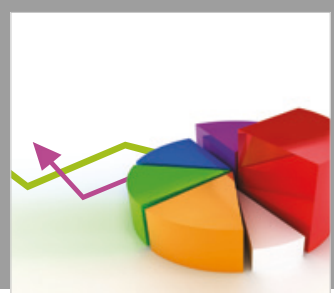

Journal of

Probability and Statistics
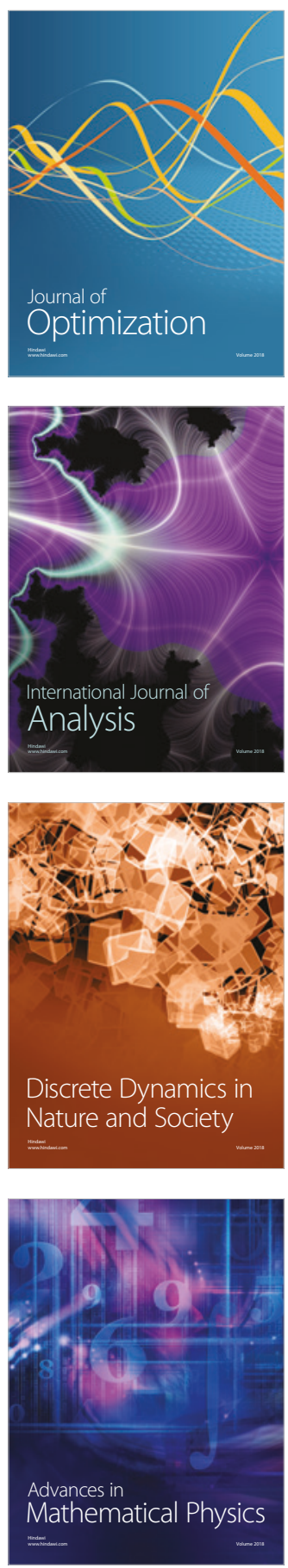